\title{
Input-To-State Stability for a Class of Switched Stochastic Nonlinear Systems by an Improved Average Dwell Time Method
}

\author{
Rongwei Guo, ${ }^{1,2}$ Ping Zhao, ${ }^{3}$ and Chenghui Zhang ${ }^{1}$ \\ ${ }^{1}$ School of Control Science and Engineering, Shandong University, Jinan 250061, China \\ ${ }^{2}$ School of Science, Qilu University of Technology, Jinan 250353, China \\ ${ }^{3}$ School of Electrical Engineering, University of Jinan, Jinan 250022, China
}

Correspondence should be addressed to Chenghui Zhang; zchui@sdu.edu.cn

Received 21 November 2013; Accepted 11 March 2014; Published 6 April 2014

Academic Editor: Ming Gao

Copyright (C) 2014 Rongwei Guo et al. This is an open access article distributed under the Creative Commons Attribution License, which permits unrestricted use, distribution, and reproduction in any medium, provided the original work is properly cited.

\begin{abstract}
This paper investigates the input-to-state stable in the mean (ISSiM) property of the switched stochastic nonlinear (SSN) systems with an improved average dwell time (ADT) method in two cases: (i) all of the constituent subsystems are ISSiM and (ii) parts of the constituent subsystems are ISSiM. First, an improved ADT method for stability of SSN systems is introduced. Then, based on that not only a new ISSiM result for SSN systems whose subsystems are ISSiM is presented, but also a new ISSiM result for such systems in which parts of subsystems are ISSiM is established. In comparison with the existing ones, the main results obtained in this paper have some advantages. Finally, an illustrative example with numerical simulation is verified the correctness and validity of the proposed results.
\end{abstract}

\section{Introduction}

Switched systems, which provide a unified framework for mathematical modeling of many physical or man-made systems, display switching features such as communication networks, manufacturing, computer synchronization, auto pilot control design, automotive engine control, traffic control, and chemical processes. The systems consist of a collection of indexed differential or difference equations and a switching signal governing the switching among them. In the past two decades, increasing attention has been paid to the analysis and synthesis of switched systems because of their significance in both theory and applications, and many significant results have been established for the stability analysis and control design of such switched systems; see [112] and references therein. Regarding the stability analysis problem, there are two famous analysis methods, that is, common Lyapunov function (CLF) method [4, 5], and multiple Lyapunov functions (MLF) method [9]. Although the CLF method is very useful in stability analysis and control design, it is difficult to be applied in practice because of the following reason: for a given switched nonlinear system, there is no general method to determine whether all subsystems share a CLF or not, even for the switched linear systems. About the MLF method, it has been proved in [9] that the switched linear systems with stable subsystems are globally asymptotically stable (GAS) if the dwell time (DT) $\tau_{d}$ is sufficiently large. Therefore, a DT method [9] is established to analyze the stability analysis and control design of the switched systems; that is, given a constant $\tau_{d}>0$, let $S_{d}\left[\tau_{d}\right]$ denote the set of all switching signals with interval between consecutive switchings being no smaller than $\tau_{d}, \tau_{d}$ is called the "dwell time". Recently, Ni et al. think it is necessary to find a minimum dwell time (MDT) $\tau_{d}^{*}$, which ensures that the switched system stays on each mode for period greater than or equal to $\tau_{d}^{*}$; the system is GAS and have obtained a new method called MDT method [13]. However, the above MDT method is only for the switched linear systems, and it is impossible to be extended to switched nonlinear systems concluding from the proofs of the results in [13]. It is well known that the ADT scheme characterizes a large class of stable switching signals than dwell time scheme. Thus, the ADT method is very important not only in practice but also in theory. Considerable attention has been paid, and many 
efforts have been done to take advantage of the ADT method to investigate the stability analysis and control design both in switched linear and nonlinear systems. In [14], we obtain an improved ADT method for the switched nonlinear systems, which have two advantages over the existing ADT methods [15-18]: one is that the conditions of the improved ADT method are less than those; the other one is that the obtained lower bound of ADT (i.e., $\tau_{a}^{*}$ ) is also smaller than those obtained by the above ADT methods.

When a control system is affected by an external input, it is important to analyze how the external input affects the system's behavior. Input-to-state stable (ISS) property [19] characterizes the continuity of state trajectories on the initial states and the external inputs, and integral input-tostate stable (iISS) property is a weaker concept introduced in [20], and the iISS property has been shown to be a natural generalization of ISS. Both ISS and iISS have been proven to be useful in the stability analysis and control design of nonlinear systems; see [21-25] and the references therein. Various extensions of the ISS property have been made for different types of dynamical systems, such as discrete time systems [21], time-delay systems [22], impulsive system [23], and switched systems [24-26]. Many works about the ISSiM of SSN systems have been done, but this problem has not been solved completely so far. Thus, investigating the ISSiM of the SSN systems is not only very important in theory but is also reasonable in practice.

In this paper, we present several new sufficient conditions under which a SSN system with an improved ADT switching signal is ISSiM, also examine the case where parts of the constituent subsystems are not ISSiM. First, we introduce an improved ADT method, and by which we present a new sufficient condition for the SSN system whose subsystems are ISSiM. Then, we obtain some new ISSiM results for such switched systems that parts of the constituent subsystems are ISSiM. Finally, an illustrative example with numerical simulation is studied using the above obtained results. The study of example shows that our analysis methods work very well in analyzing the ISSiM of SSN systems.

The rest of the paper is organized as follows. Section 2 introduces some notations and preliminary results which are used in this paper. Section 3 presents the main results of this paper. In Section 4, an illustrative example with numerical simulation is given to support our new results, which is followed by the conclusion in Section 5 .

\section{Notations and Preliminary Results}

Throughout this paper, $\mathbb{R}_{+}$denotes the set of all nonnegative real numbers; $\mathbb{R}^{n}$ and $\mathbb{R}^{n \times m}$ denote $n$-dimensional real space and $n \times m$ dimensional real matrix space, respectively. For vector $x \in \mathbb{R}^{n},|x|$ denotes the Euclidean norm; that is, $|x|=\left(\sum_{i=1}^{n} x_{i}^{2}\right)^{1 / 2}$. All the vectors are column vectors unless otherwise specified; the transpose of vectors and matrices are denoted by superscript $T ; \mathscr{C}^{i}$ denotes all the $i$ th continuous differential functions. A function $\varphi(u)$ is said to belong to the class $\mathscr{K}$ if $\varphi \in \mathscr{C}\left(\mathbb{R}_{+}, \mathbb{R}_{+}\right), \varphi(0)=0$ and $\varphi(u)$ is strictly increasing in $u . \mathscr{K}_{\infty}$ is the subset of $\mathscr{K}$ functions that are unbounded.

Consider the following SSN systems:

$$
d x=f_{\sigma}(x, u) d t+g_{\sigma}(x, u) d w, \quad t \geq 0,
$$

where $x \in \mathbb{R}^{n}$ and $u \in \mathscr{L}_{\infty}^{m}$ are the system state and input, respectively; $\mathscr{L}_{\infty}^{m}$ denotes the set of all the measurable and locally essentially bounded input $u \in \mathbb{R}^{m}$ on $[0, \infty)$ with norm

$$
\|u(t)\|=\inf _{\mathscr{A} \subset \Omega, P(\mathscr{A})=0} \sup \{|u(t, \omega)|: \omega \in \Omega \backslash \mathscr{A}\} .
$$

$w$ is an $r$-dimensional independent standard Wiener process (or Brownian motion); $\sigma(\cdot):[0, \infty) \rightarrow \mathscr{I}(\mathscr{I}$ is the index set, maybe infinite) is the switching path (or law, signal) and is right-hand continuous and piecewise constant on $t$. More specifically, we impose restrictions on the set of admissible switching signals by defining the set

$$
D_{T}=\left\{\sigma(t): t_{k+1}-t_{k} \geq T\right\}
$$

where $t_{k}$ are the commutation instants and $T \geq 0$. For every $i \in \mathscr{I}, f_{i}: \mathbb{R}^{n} \times \mathbb{R}^{m} \rightarrow \mathbb{R}^{n}, g_{i}: \mathbb{R}^{n} \times \mathbb{R}^{m} \rightarrow$ $\mathbb{R}^{n \times r}$ is continuous, uniformly locally Lipschitz, and satisfies $f_{i}(0,0)=g_{i}(0,0)=0$; initial data $x_{0} \in \mathbb{R}^{n}$. For an arbitrary matrix $D$, we define $|D|=\left[\lambda_{M}\left(D^{T} D\right)\right]^{1 / 2}$, where $\lambda_{M}$ denotes the largest eigenvalue of $D^{T} D$.

For any given $V(x) \in C^{2}\left(\mathbb{R}^{n} ; \mathbb{R}_{+}\right)$, associated with the SSN system (1), we define the differential operator $\mathscr{L}$ to every $i \in \mathscr{I}$ as follows:

$$
\mathscr{L} V=\frac{\partial V}{\partial x} f_{i}(x, u)+\frac{1}{2} \operatorname{Tr}\left\{g_{i}^{T}(x, u) \frac{\partial^{2} V}{\partial x^{2}} g_{i}(x, u)\right\} .
$$

With the development of this paper, we first present some definitions.

Definition 1 (see [15]). For any switching signal $\sigma(t)$ and any $t \geq \tau$, let $N_{\sigma}(\tau, t)$ denote the number of switching of $\sigma(t)$ over the interval $[\tau, t)$ satisfying

$$
N_{\sigma}(\tau, t) \leq N_{0}+\frac{t-\tau}{\tau_{a}},
$$

where $\tau_{a}$ is called average dwell time and $N_{0}$ denotes the chatter bound.

Definition 2 (see [27]). The SSN system (1) is ISSiM if there exist $\beta \in \mathscr{K} \mathscr{L}$ and $\alpha, \gamma \in \mathscr{K}_{\infty}$, such that for any $u \in \mathbb{R}^{m}$, $x_{0} \in \mathbb{R}^{n}$, we have

$$
E[\alpha(|x(t)|)] \leq \beta\left(\left|x_{0}\right|, t\right)+\gamma\left(\|u\|_{[0, t)}\right), \quad \forall t \geq 0 .
$$

The SSN system (1) is $e^{\lambda t}$-weighted ISSiM for some $\lambda>0$; if there exist $\alpha_{1}, \alpha_{2}, \gamma \in \mathscr{K}_{\infty}$ such that for any $u \in \mathbb{R}^{m}, x_{0} \in$ $\mathbb{R}^{n}$, we have

$$
\begin{aligned}
& e^{\lambda t} E\left[\alpha_{1}(|x(t)|)\right] \\
& \quad \leq \alpha_{2}\left(\left|x_{0}\right|\right)+\sup _{s \in[0, t)}\left\{e^{\lambda s} \gamma(\|u(s)\|)\right\}, \quad \forall t \geq 0 .
\end{aligned}
$$


The SSN system (1) is $e^{\lambda t}$-weighted integral ISSiM for some $\lambda>0$; if there exist $\alpha_{1}, \alpha_{2}, \gamma \in \mathscr{K}_{\infty}$ such that for any $u \in \mathbb{R}^{m}, x_{0} \in \mathbb{R}^{n}$, we have

$$
\begin{aligned}
& e^{\lambda t} E\left[\alpha_{1}(|x(t)|)\right] \\
& \quad \leq \alpha_{2}\left(\left|x_{0}\right|\right)+\int_{0}^{t} e^{\lambda \tau} \gamma(\|u(\tau)\|) d \tau, \quad \forall t \geq 0 .
\end{aligned}
$$

In [28], we have obtained an improved ADT method to investigate the stability of the SSN system (1) with $u \equiv 0$ in two cases: one is that all constituent subsystems are globally exponentially stable in the mean (GASiM) and the other is that some constituent subsystems are GASiM, while some of them are not GASiM. We introduce those results in the following.

Lemma 3 (see [28]). For the SSN system (1) with $u \equiv 0$, if there exist $\mathscr{C}^{1}$ functions $V_{i}: \mathbb{R}^{n} \rightarrow[0, \infty), i \in \mathscr{J}$, and functions $\alpha, \beta \in \mathscr{K}_{\infty}$ such that

$$
\begin{gathered}
\alpha(|x|) \leq V_{i}(x) \leq \beta(|x|), \quad \forall i \in \mathscr{I}, \\
\left.\mathscr{L} V_{i}(x)\right|_{(i)} \leq-\lambda_{i} V_{i}(x),
\end{gathered}
$$

where $\lambda_{i}>0, i \in \mathscr{I}$, then the SSN system (1) with $u \equiv 0$ is GASiM under any switching signal with ADT:

$$
\tau_{a}>\tau_{a}^{*}=\frac{a}{\lambda_{\min }}
$$

where

$$
a=\ln \mu, \quad \mu=\sup _{x \neq 0} \frac{\beta(|x(t)|)}{\alpha(|x(t)|)}, \quad \lambda_{\min }=\min _{i \in \mathscr{I}} \lambda_{i} .
$$

Remark 4. If $\mu=1$, which implies that $V_{i}(x) \equiv V(x)$, $i \in \mathscr{I}, V(x)$ is a CLF for the SSN system (1) with $u \equiv 0$, and thus this system is GASiM under arbitrary switching. It is also noted that the ADT method proposed in [15-18] needs the conditions (9)-(10) and an additional condition as " $V_{i}(x) \leq \mu V_{j}(x), \mu \geq 1, i \neq j, i, j \in \mathscr{I}$ ". Comparing Lemma 3 with the corresponding results in $[15,16]$, Lemma 3 needs fewer conditions and thus can be applied to a wider range of systems.

Moreover, it is noted that the above $\alpha$ and $\beta$ in (9) should have the same order, and which can ensure that $\mu$ exists. Furthermore, if $V_{i}(x)=x^{T} P_{i} x, P_{i}>0$, then inequality (9) becomes

$$
\alpha_{i}|x|^{2} \leq V_{i}(x) \leq \beta_{i}|x|^{2}
$$

and $\mu$ is given as

$$
\mu=\max _{i \in \mathscr{I}} \frac{\beta_{i}}{\alpha_{i}}
$$

For this case, if we use the ADT method in [15-18], we can get

$$
\mu^{\prime}=\max _{i, j \in \mathscr{I}} \frac{\beta_{i}}{\alpha_{j}}
$$

Obviously, $\mu \leq \mu^{\prime}$.
In particular, for the switched linear systems, the lower bound $\mathrm{ADT} \tau_{a}^{*}$ obtained by Lemma 3 is smaller than the lower bound $\mathrm{ADT} \tau_{a}^{\prime}$ obtained in [15]; that is,

$$
\tau_{a}^{*}=\max _{i \in \mathscr{I}}\left\{\frac{\lambda_{\max }\left(P_{i}\right)}{\lambda_{\min }\left(P_{i}\right) \lambda_{\min }}\right\} \leq \max _{i, j \in \mathscr{I}}\left\{\frac{\lambda_{\max }\left(P_{j}\right)}{\lambda_{\min }\left(P_{i}\right) \lambda_{\min }}\right\}=\tau_{a}^{\prime},
$$

where $V_{i}(x)=x^{T} P_{i} x$ with $P_{i}>0$ is the Lyapunov function for the $i$ th subsystem, $i \in \mathscr{I}$.

This improved ADT method can also be extended to analyze the stability of the SSN system (1) with $u \equiv 0$ in which both stable and unstable subsystems coexist. For the switching signal $\sigma(t)$ and any $t>\tau$, we let $T^{u}(\tau, t)$ (resp., $T^{\mathcal{S}}(\tau, t)$ ) denote the total activation time of the unstable subsystems (resp., the stable subsystems) on interval $[\tau, t)$. Then, we let $\mathscr{I}=\mathscr{I}_{s} \cup \mathscr{I}_{u}$ such that $\mathscr{I}_{s} \cap \mathscr{I}_{u}=\emptyset$ and introduce a switching law form [16].

(S1) Determine the $\sigma(t)$ satisfying $T^{s}\left(t_{0}, t\right) / T^{u}\left(t_{0}, t\right) \geq$ $\left(\lambda_{u}+\lambda^{*}\right) /\left(\lambda_{s}-\lambda^{*}\right)$ holds for any $t>t_{0}$, where $\lambda^{*} \in\left(0, \lambda_{s}\right) ; \lambda_{u}$ and $\lambda_{s}$ are given as (19).

Next, we introduce the result in the following.

Lemma 5 (see [28]). Consider the SSN system (1) with $u \equiv 0$; if there exist $\mathscr{C}^{1}$ functions $V_{i}(x): \mathbb{R}^{n} \rightarrow[0, \infty), i \in \mathscr{I}$, and functions $\alpha, \beta \in \mathscr{K}_{\infty}$ such that (9), and

$$
\begin{aligned}
& \left.\mathscr{L} V_{i}(x)\right|_{(i)} \leq \lambda_{i} V_{i}(x), \quad i \in \mathscr{I}_{u}, \\
& \left.\mathscr{L} V_{i}(x)\right|_{(i)} \leq-\lambda_{i} V_{i}(x), \quad i \in \mathscr{I}_{s},
\end{aligned}
$$

where $\lambda_{i}>0, i \in \mathscr{I}$, then under the switching law S1, the switched system (1) with $u \equiv 0$ is GASiM for any switching signal with ADT:

$$
\tau_{a}>\tau_{a}^{*}=\frac{a}{\lambda^{*}}
$$

where a is given as (12), and $\lambda^{*} \in\left(0, \lambda_{s}\right)$ is an arbitrarily chosen number,

$$
\lambda_{s}=\max _{i \in \mathscr{I}_{s}} \lambda_{i}, \quad \lambda_{u}=\max _{i \in \mathscr{I}_{u}} \lambda_{i}
$$

Remark 6. Similar to Remark 4, comparing Lemma 5 with the corresponding existing results in $[15,16]$, Lemma 5 needs fewer conditions, and thus Lemma 5 is really an improvement of the existing results.

\section{Main Results}

3.1. All Subsystems Are ISSiM. In this section, we first investigate the ISSiM stability of the SSN system (1) in which all constituent subsystems are ISSiM. According to Lemma 3, we obtain the following result. 
Theorem 7. Considering the SSN system (1), if there exist $\mathscr{C}^{1}$ functions $V_{i}: \mathbb{R}^{n} \rightarrow[0, \infty), i \in \mathscr{I}$, functions $\bar{\alpha}_{1}, \bar{\alpha}_{2}, \bar{\gamma} \in \mathscr{K}_{\infty}$ and number $\lambda_{0}>0$ such that for all $\xi \in \mathbb{R}^{n}, \eta \in \mathbb{R}^{\mathscr{L}}$,

$$
\begin{gathered}
\bar{\alpha}_{1}(|\xi|) \leq V_{i}(\xi) \leq \bar{\alpha}_{2}(|\xi|), \\
\left.\mathscr{L} V_{i}(\xi)\right|_{(i)} \leq-\lambda_{0} V_{i}(\xi)+\bar{\gamma}(|\eta|), \quad i \in \mathcal{F},
\end{gathered}
$$

then

(I) if $\tau_{a}>\tau_{a}^{*}=a / \lambda_{0}$, then the SSN system (1) is ISSiM;

(II) if $\tau_{a}>\tau_{a}^{*}=a /\left(\lambda_{0}-\lambda-\delta\right)$, for some $\lambda \in\left(0, \lambda_{0}-\delta\right)$, where $\delta>0$, then the SSN system (1) is $e^{\lambda t}$-weighted ISSiM;

(III) if $\tau_{a}>\tau_{a}^{*}=a /\left(\lambda_{0}-\lambda\right)$, for some $\lambda \in\left(0, \lambda_{0}\right)$, then the SSN system (1) is $e^{\lambda t}$-weighted integral ISSiM,

where $a$ is given as (12).

Proof. For notational brevity, define $G_{a}^{b}(\lambda)=$ $\int_{a}^{b} e^{\lambda s} \bar{\gamma}(\|u(s)\|) d s$. Let $T>0$ be an arbitrary time. Denote by $t_{1}, \ldots, t_{N_{\sigma}(0, T)}$ the switching times on the interval $(0, T)$ (by convention, $\left.t_{0}:=0, t_{N_{\sigma}(0, T)}:=T\right)$. Consider the piecewise continuously differentiable function

$$
W(s):=e^{\lambda_{0} s} V_{\sigma(s)}(x(s)) .
$$

On each interval $\left[t_{i}, t_{i+1}\right)$, the switching signal is constant. Consider

$$
\begin{aligned}
W(t)= & W\left(t_{i}\right)+\int_{t_{i}}^{t} e^{\lambda_{0} s} \frac{\partial V_{\sigma\left(t_{i}\right)}}{\partial x} g_{\sigma(s)}(s, x(s)) d w(s) \\
& +\int_{t_{i}}^{t} e^{\lambda_{0} s}\left(\mathscr{L} V_{\sigma(s)}(x(s))+\lambda_{0} V_{\sigma(s)}(x(s))\right) d s .
\end{aligned}
$$

If $t$ is replaced by $t_{r}=\min \left\{t, \tau_{r}\right\}$ in the above, where $\tau_{r}=\inf \{s \geq 0:|x(s)| \geq r\}$, then the stochastic integral (first integral) in (23) defines a martingale (with $r$ fixed and $t$ varying), not just a local martingale. Thus, on taking expectations in (23) with $t_{r}$ in place of $t$ and then using (21) on the right, we get

$$
E W\left(t_{r}\right) \leq E W\left(t_{i}\right)+E\left[\int_{t_{i}}^{t_{r}} e^{\lambda_{0} s} \bar{\gamma}(\|u(s)\|) d s\right] .
$$

On letting $r \rightarrow \infty$ and using Fatou's Lemma on the left and monotone convergence on the right, we conclude

$$
E W(t) \leq E W\left(t_{i}\right)+\int_{t_{i}}^{t} e^{\lambda_{0} s} \bar{\gamma}(\|u(s)\|) d s
$$

According to inequality (20), we obtain

$$
\begin{aligned}
& E \bar{\alpha}_{1}\left(\left|x_{T}\right|\right) \\
& \leq E V_{\sigma\left(t_{k}\right)}\left(x_{T}\right) \leq e^{-\lambda_{0}\left(T-t_{k}\right)} E V_{\sigma\left(t_{k}\right)}\left(x_{k}\right) \\
& +e^{-\lambda_{0} T} G_{t_{k}}^{T}\left(\lambda_{0}\right) \\
& \leq e^{-\lambda_{0}\left(T-t_{k}\right)} E \bar{\alpha}_{2}\left(\left|x_{k}\right|\right)+e^{-\lambda_{0} T} G_{t_{k}}^{T}\left(\lambda_{0}\right) \\
& =e^{-\lambda_{0}\left(T-t_{k}\right)} \frac{E \bar{\alpha}_{2}\left(\left|x_{k}\right|\right)}{E \bar{\alpha}_{1}\left(\left|x_{k}\right|\right)} E \bar{\alpha}_{1}\left(\left|x_{k}\right|\right)+e^{-\lambda_{0} T} G_{t_{k}}^{T}\left(\lambda_{0}\right) \\
& \leq \mu e^{-\lambda_{0}\left(T-t_{k}\right)} E \bar{\alpha}_{1}\left(\left|x_{k}\right|\right)+e^{-\lambda_{0} T} G_{t_{k}}^{T}\left(\lambda_{0}\right) \\
& \leq \mu^{2} e^{-\lambda_{0}\left(T-t_{k-1}\right)} E \bar{\alpha}_{1}\left(\left|x_{k-1}\right|\right)+\mu e^{-\lambda_{0} T} G_{t_{k-1}}^{t_{k}}\left(\lambda_{0}\right) \\
& +e^{-\lambda_{0} T} G_{t_{k}}^{T}\left(\lambda_{0}\right) \\
& \vdots \\
& \leq \mu^{N_{\sigma(t)}\left(t_{0}, T\right)+1} e^{-\lambda_{0}\left(T-t_{0}\right)} E \bar{\alpha}_{1}\left(\left|x_{0}\right|\right) \\
& +e^{-\lambda_{0} T} \sum_{j=0}^{N_{\sigma(t)}\left(t_{0}, T\right)} \mu^{N_{\sigma(t)}\left(t_{0}, T\right)-j} G_{t_{j}}^{t_{j+1}}\left(\lambda_{0}\right) \\
& =\mu e^{a N_{\sigma(t)}\left(t_{0}, T\right)-\lambda_{0}\left(T-t_{0}\right)} E \bar{\alpha}_{1}\left(\left|x_{0}\right|\right) \\
& +\sum_{j=0}^{N_{\sigma(t)}\left(t_{0}, T\right)} \mu^{N_{\sigma(t)}\left(t_{0}, T\right)-j} G_{t_{j}}^{t_{j+1}}\left(\lambda_{0}\right) \\
& \leq \mu^{1+N_{0}} e^{\left(a / \tau_{a}-\lambda_{0}\right)\left(T-t_{0}\right)} E \bar{\alpha}_{1}\left(\left|x_{0}\right|\right) \\
& +\sum_{j=0}^{N_{\sigma(t)}\left(t_{0}, T\right)} \mu^{N_{\sigma(t)}\left(t_{0}, T\right)-j} G_{t_{j}}^{t_{j+1}}\left(\lambda_{0}\right) .
\end{aligned}
$$

For every $\delta \in\left[0, \lambda_{0}-\lambda-a / \tau_{a}^{*}\right]$, that is, $\tau_{a}^{*} \geq a /\left(\lambda_{0}-\lambda-\delta\right)$, where $\lambda>0$,

$$
\begin{gathered}
G_{t_{j}}^{t_{j+1}}\left(\lambda_{0}\right) \leq e^{\left(\lambda_{0}-\lambda-\delta\right) t_{k+1}} G_{t_{j}}^{t_{j+1}}(\lambda+\delta), \\
\mu^{N_{\sigma(t)}\left(t_{0}, T\right)-j} \leq \mu^{1+N_{0}} e^{\left(\lambda_{0}-\lambda-\delta\right)\left(T-t_{j+1}\right)} .
\end{gathered}
$$

Therefore,

$$
\begin{aligned}
& \sum_{j=0}^{N_{\sigma(t)}\left(t_{0}, T\right)} \mu^{N_{\sigma(t)}\left(t_{0}, T\right)-j} G_{t_{j}}^{t_{j+1}}\left(\lambda_{0}\right) \\
& \quad \leq \mu^{1+N_{0}} e^{\left(\lambda_{0}-\lambda-\delta\right) T} G_{t_{0}}^{T}(\lambda+\delta) .
\end{aligned}
$$

Substituting inequality (28) to inequality (26), we get

$$
\begin{array}{r}
E \bar{\alpha}_{1}\left(\left|x_{T}\right|\right) \leq \mu^{1+N_{0}}\left[e^{\left(a / \tau_{a}-\lambda_{0}\right)\left(T-t_{0}\right)} \bar{\alpha}_{1}\left(\left|x_{0}\right|\right)\right. \\
\left.+e^{-(\lambda+\delta) T} G_{t_{0}}^{T}(\lambda+\delta)\right],
\end{array}
$$


that is,

$$
\begin{array}{r}
e^{(\lambda+\delta) T} E \bar{\alpha}_{1}\left(\left|x_{T}\right|\right) \leq \mu^{1+N_{0}}\left[e^{\lambda+\delta t_{0}} e^{\left(\left(a / \tau_{a}\right)-\lambda_{0}+\lambda+\delta\right)\left(T-t_{0}\right)}\right. \\
\left.\times \bar{\alpha}_{1}\left(\left|x_{0}\right|\right)+G_{t_{0}}^{T}(\lambda+\delta)\right] \\
=C\left[e^{(\lambda+\delta) t_{0}} e^{\left(a / \tau_{a}-\lambda_{0}+\lambda+\delta\right)\left(T-t_{0}\right)}\right. \\
\left.\times \bar{\alpha}_{1}\left(\left|x_{0}\right|\right)+G_{t_{0}}^{T}(\lambda+\delta)\right],
\end{array}
$$

where $C=\mu^{1+N_{0}}$.

For inequality (30), if $\delta=0$, then

$$
\begin{array}{rl}
e^{\lambda T} & E \bar{\alpha}_{1}\left(\left|x_{T}\right|\right) \\
\leq & C e^{\lambda t_{0}} e^{\left(a / \tau_{a}-\lambda_{0}+\lambda\right)\left(T-t_{0}\right)} \bar{\alpha}_{1}\left(\left|x_{0}\right|\right)+C G_{t_{0}}^{T}(\lambda) \\
= & C e^{\lambda t_{0}} e^{\left(a / \tau_{a}-\lambda_{0}+\lambda\right)\left(T-t_{0}\right)} \bar{\alpha}_{1}\left(\left|x_{0}\right|\right) \\
& +C \int_{t_{0}}^{T} e^{\lambda s} \bar{\gamma}(|u(s)|) d s .
\end{array}
$$

For inequality (31), if $\tau_{a}>a /\left(\lambda_{0}-\lambda\right)$, we have property (8) with

$$
\begin{gathered}
\alpha_{1}\left(\left|x_{T}\right|\right):=\bar{\alpha}_{1}\left(\left|x_{T}\right|\right), \\
\alpha_{2}\left(\left|x_{0}\right|\right):=C e^{\lambda t_{0}} e^{\left(a / \tau_{a}-\lambda_{0}+\lambda\right)\left(T-t_{0}\right)} \bar{\alpha}_{1}\left(\left|x_{0}\right|\right) .
\end{gathered}
$$

Note that

$$
G_{t_{0}}^{T}(\lambda+\delta) \leq \frac{1}{\lambda+\delta-\bar{\lambda}} e^{(\lambda+\delta-\bar{\lambda}) T} \sup _{\tau \in[0, T)}\left\{e^{\bar{\lambda} \tau} \bar{\gamma}(|u(\tau)|)\right\} .
$$

Substituting inequality (33) to inequality (30), we obtain

$$
\begin{aligned}
& e^{(\lambda+\delta) T} E \bar{\alpha}_{1}\left(\left|x_{T}\right|\right) \\
& \leq C e^{(\lambda+\delta) t_{0}} e^{\left(a / \tau_{a}-\lambda_{0}+\lambda+\delta\right)\left(T-t_{0}\right)} \bar{\alpha}_{1}\left(\left|x_{0}\right|\right) \\
& \quad+C \frac{1}{\lambda+\delta-\bar{\lambda}} e^{(\lambda+\delta-\bar{\lambda}) T} \sup _{\tau \in[0, T)}\left\{e^{\bar{\lambda} \tau} \bar{\gamma}(|u(\tau)|)\right\} .
\end{aligned}
$$

For inequality (34), if $\delta \neq 0, \bar{\lambda}=\lambda$, we get

$$
\begin{aligned}
e^{\lambda T} E & \bar{\alpha}_{1}\left(\left|x_{T}\right|\right) \\
\leq & C e^{\lambda t_{0}} e^{\left(a / \tau_{a}-\lambda_{0}+\lambda+\delta\right)\left(T-t_{0}\right)} \\
& \times E \bar{\alpha}_{1}\left(\left|x_{T}\right|\right)+C \frac{1}{\delta} e^{\delta T} \sup _{\tau \in[0, T)}\left\{e^{\bar{\lambda} \tau} \bar{\gamma}(|u(\tau)|)\right\} .
\end{aligned}
$$

For inequality (35), if $\tau_{a}>a /\left(\lambda_{0}-\lambda-\delta\right)$, we have property (7) with

$$
\begin{gathered}
\alpha_{1}\left(\left|x_{T}\right|\right):=e^{-\delta T} \frac{\delta}{C} \bar{\alpha}_{1}\left(\left|x_{T}\right|\right) \\
\alpha_{2}\left(\left|x_{0}\right|\right):=e^{-\delta T} \delta e^{\lambda t_{0}} e^{\left(a / \tau_{a}-\lambda_{0}+\lambda+\delta\right)\left(T-t_{0}\right)} \bar{\alpha}_{1}\left(\left|x_{0}\right|\right) .
\end{gathered}
$$

For inequality (34), if $\bar{\lambda}=\delta=0$, we obtain that

$$
\begin{aligned}
\bar{\alpha}_{1}\left(\left|x_{T}\right|\right) \leq & C e^{\lambda t_{0}} e^{\left(a / \tau_{a}-\lambda_{0}\right)\left(T-t_{0}\right)} \bar{\alpha}_{1}\left(\left|x_{T}\right|\right) \\
& +C \frac{1}{\lambda} \sup _{\tau \in[0, T)}\{\bar{\gamma}(|u(\tau)|)\} .
\end{aligned}
$$

For inequality (37), if $\tau_{a}>a /\left(\lambda_{0}-\lambda\right)$, we have property (6) with

$$
\alpha_{1}\left(\left|x_{T}\right|\right):=C e^{\lambda t_{0}} e^{\left(a / \tau_{a}-\lambda_{0}\right)\left(T-t_{0}\right)} \bar{\alpha}_{1}\left(\left|x_{T}\right|\right), \quad \gamma:=C \frac{1}{\lambda} \bar{\gamma} .
$$

Remark 8. It should be pointed out that the result proposed in [25] needs conditions (20)-(21) and an additional condition as " $V_{i}(x) \leq \mu V_{j}(x), \mu \geq 1, i \neq j, i, j \in \mathscr{I}^{\prime}$. Comparing Theorem 7 with the existing result in [25], Theorem 7 needs fewer conditions and thus is an improvement of the existing result.

3.2. Some Subsystems Are Not ISSiM. In the next, we consider the SSN system (1) in which both ISSiM and not ISSiM subsystems coexist. Similarly, for the switching signal $\sigma(t)$ and any $t>\tau$, we let $T^{\mathcal{u}}(\tau, t)$ (resp., $\left.T^{\mathcal{S}}(\tau, t)\right)$ denote the total activation time of the not ISSiM subsystems (resp., the ISSiM subsystems) on interval $[\tau, t)$.

According to Lemma 5, we give the following result.

Theorem 9. Considering the SSN system (1), if there exist $\mathscr{C}^{1}$ functions $V_{i}: \mathbb{R}^{n} \rightarrow[0, \infty), i \in \mathscr{I}$, and functions $\alpha_{1}, \alpha_{2}$, $\varphi_{1} \in \mathscr{K}_{\infty}$, constants $\lambda_{s}, \lambda_{u}>0$ such that (20) for all $x \in \mathbb{R}^{n}$, and furthermore, the following inequalities hold:

$$
\begin{aligned}
|x| & \geq \varphi_{1}(u) \\
& \Longrightarrow \begin{cases}\left.\mathscr{L} V_{i}(x)\right|_{(i)} \leq \lambda_{u} V_{i}(x), & i \in \mathscr{I}_{u}, \\
\left.\mathscr{L} V_{i}(x)\right|_{(i)} \leq-\lambda_{s} V_{i}(x), & i \in \mathscr{I}_{s} .\end{cases}
\end{aligned}
$$

Then, under the switching law S1, the SSN system (1) is ISSiM for any switching signal with ADT:

$$
\tau_{a}>\tau_{a}^{*}=\frac{a}{\lambda^{*}},
$$

where $a$ is given as (12), and $\lambda^{*} \in\left(0, \lambda_{s}\right)$ is an arbitrarily chosen number; $\lambda_{s}$ and $\lambda_{u}$ are given as (19).

Proof. Let $t_{0} \geq 0$ be arbitrary. For $t \geq t_{0}$, define $\nu(t):=$ $\varphi_{1}\left(\|u\|_{\left[t_{0}, t\right]}\right)$ and $\xi(t):=\alpha_{1}^{-1}\left(\mu^{N_{0}} \alpha_{1}(\nu(t))\right)$, where $N_{0}$ comes from (5). Furthermore, define the balls around the origin $B_{\nu}(t):=\{x:|x| \leq \nu(t)\}, B_{\xi}(t):=\{x:|x| \leq \xi(t)\}$. Note that $\nu$ and thus also $\xi$ are nondecreasing functions of time, and thus the balls $B_{v}$ and $B_{\xi}$ have nondecreasing volume.

If $|x(t)| \geq v(t) \geq \varphi_{1}(\|u(t)\|)$ during some time interval $t \in\left[t^{\prime}, t^{\prime \prime}\right]$, then $x(t)$ can be bounded above by

$$
\begin{aligned}
E|x(t)| & \leq E \alpha_{1}^{-1}\left(\mu^{N_{0}} e^{-\lambda^{*}\left(t-t^{\prime}\right)} \alpha_{1}\left(\left|x\left(t^{\prime}\right)\right|\right)\right) \\
& :=\beta\left(\left|x\left(t^{\prime}\right)\right|, t-t^{\prime}\right)
\end{aligned}
$$

for some $\lambda^{*} \in\left(0, \lambda_{s}\right)$. 
In fact, on any interval $\left[\tau_{i}, \tau_{i+1}\right) \cap\left[t^{\prime}, t^{\prime \prime}\right]$, according to (39), we arrive at

$$
E \alpha_{1}(|x(t)|) \leq e^{a N_{\sigma}\left(t^{\prime}, t\right)+\lambda_{u} T^{u}\left(t^{\prime}, t\right)-\lambda_{s} T^{s}\left(t^{\prime}, t\right)} E \alpha_{1}\left(\left|x\left(t^{\prime}\right)\right|\right)
$$

Then, according to (5) and the switching law S1, we conclude from (42) that

$$
\begin{aligned}
E \alpha_{1}(|x(t)|) & \leq e^{a N_{0}} e^{\left(a / \tau_{a}-\lambda^{*}\right)\left(t-t^{\prime}\right)} E \alpha_{1}\left(\left|x\left(t^{\prime}\right)\right|\right) \\
& =\mu^{N_{0}} e^{\left(a / \tau_{a}-\lambda^{*}\right)\left(t-t^{\prime}\right)} E \alpha_{1}\left(\left|x\left(t^{\prime}\right)\right|\right) .
\end{aligned}
$$

Thus, if $\tau_{a}>\tau_{a}^{*}=a / \lambda^{*}$, we can get (41).

Denote the first time when $x(t) \in B_{v}(t)$ by $\breve{t}_{1}$; that is, $\breve{t}_{1}:=$ $\inf \left\{t \geq t_{0}:|x(t)| \leq v(t)\right\}$. For $t_{0} \leq t \leq \breve{t}_{1}$, according to (41), we obtain

$$
E|x(t)| \leq \beta\left(\left|x_{0}\right|, t-t_{0}\right) .
$$

If $\breve{t}_{1}=\infty$, which only can happen if $v(t) \equiv 0$, that is, the input $u \equiv 0$ for all times, then the SSN system (1) is ISSiM. Hence in the following we assume that $\breve{t}_{1}<\infty$.

For $t>\breve{t}_{1}, x(t)$ can be bounded above in terms of $\nu(t)$. Namely, let $\widehat{t}_{1}:=\inf \left\{t>\breve{t}_{1}:|x(t)|>v(t)\right\}$. If this is an empty set, let $\widehat{t}_{1}:=\infty$. Clearly, for all $t \in\left[\breve{t}_{1}, \breve{t}_{2}\right)$, it holds that $|x(t)| \leq$ $\nu(t) \leq \xi(t)$. For the case that $\widehat{t}_{1}<\infty$, due to continuity of $x(\cdot)$ and monotonicity for $\nu(t)$, it holds that $\left|x\left(\widehat{t}_{1}\right)\right|=\nu\left(\widehat{t}_{1}\right)$. Furthermore, for all $\tau>\widehat{t}_{1}$, if $|x(\tau)|>\nu(\tau)$, define

$$
\widehat{t}:=\sup \{t<\tau:|x(t)| \leq v(t)\}
$$

which can be interpreted as the previous exit time of the trajectory $x(t)$ from the ball $B_{v}$. Again, due to the same argument as above, one obtains that $E\left|x\left(\hat{t}_{1}\right)\right|=v\left(\hat{t}_{1}\right)$. But then, according to (41), it holds that

$$
\begin{aligned}
E|x(\tau)| & \leq \beta(\nu(\widehat{t}), \tau-\widehat{t})=E \alpha_{1}^{-1}\left(\mu^{N_{0}} e^{-\lambda^{*}(\tau-\widehat{t})} \alpha_{1}(\nu(\widehat{t}))\right) \\
& \leq E \alpha_{1}^{-1} \mu^{N_{0}} E \alpha_{1}(\nu(\widehat{t}))=\xi(\widehat{t}) \leq \xi(\tau) .
\end{aligned}
$$

Summarizing the above, for all $t \geq \breve{t}_{1}$, it holds that

$$
\begin{aligned}
E|x(t)| & \leq \xi(t)=E \alpha_{1}^{-1}\left(\mu^{N_{0}} \alpha_{1}\left(\varphi_{1}\left(\|u\|_{\left[t_{0}, t\right]}\right)\right)\right) \\
& \leq E \alpha_{1}^{-1} \mu^{N_{0}} \alpha_{1}\left(2 \varphi_{1}\left(\|u\|_{\left[t_{0}, t\right]}\right)\right):=\gamma_{1}\left(\|u\|_{\left[t_{0}, t\right]}\right) .
\end{aligned}
$$

Combining (44) and (47), we obtain that

$$
E|x(t)| \leq \beta\left(\left|x_{0}\right|, t-t_{0}\right)+\gamma_{1}\left(\|u\|_{\left[t_{0}, t\right]}\right)
$$

for all $t \geq t_{0}$, which means the SSN system (1) is ISSiM and also completes the proof.

\section{An Illustrative Example}

In this section, we give an illustrative example to show how to use the obtained results to analyze the ISSiM stability of SSN systems.

Example 1. Consider the following SSN system:

$$
d x=f_{i}(x, u) d t+g_{i}(x) d w(t),
$$

where $i \in \mathscr{I}=\{1,2\}, w$ is an $r$-dimensional standard Brownian motion, and

$$
\begin{gathered}
f_{1}(x, u)=\left(\begin{array}{c}
-x_{1}-x_{1} x_{2}^{2}-x_{1} \sin ^{2} t \\
x_{1}^{2} x_{2}-3 x_{2}-x_{2} \cos ^{2} t
\end{array}\right), \\
f_{2}(x, u)=\left(\begin{array}{c}
2 x_{1}+2 x_{2} \\
x_{1}+3 x_{2}
\end{array}\right), \\
g_{1}(x)=\left(\begin{array}{c}
\frac{1}{2} x_{1}-\frac{1}{2} x_{2} \\
-\frac{1}{2} x_{1}+\frac{1}{2} x_{2}
\end{array}\right), \\
g_{2}(x)=\left(\begin{array}{c}
-\frac{1}{2} x_{1} \\
\frac{\sqrt{3}}{2} x_{2}
\end{array}\right) .
\end{gathered}
$$

It is easy to know that $V(x)=x^{T} x$ is a CLF for the system (49), and

$$
\begin{aligned}
\left.\mathscr{L} V_{1}(x)\right|_{(1)} & =\frac{\partial V}{\partial x} f_{i}(x)+\frac{1}{2} \operatorname{Tr}\left\{g_{i}^{T}(x) \frac{\partial^{2} V}{\partial x^{2}} g_{i}(x)\right\} \\
& =-2 x_{1}^{2}-6 x_{2}^{2} \leq-2 V_{1}(x), \\
\left.\mathscr{L} V_{2}(x)\right|_{(2)} & =\frac{\partial V}{\partial x} f_{i}(x)+\frac{1}{2} \operatorname{Tr}\left\{g_{i}^{T}(x) \frac{\partial^{2} V}{\partial x^{2}} g_{i}(x)\right\} \\
& =2 x_{1}^{2}+4 x_{1} x_{2}+3 x_{2}^{2} \leq 5 V_{2}(x) .
\end{aligned}
$$

According to the above results, we obtain that $\lambda_{u}=5, \lambda_{s}=2$ and $a=0$. Therefore, the lower bound $\operatorname{ADT} \tau_{a}^{*}=0$; that is, the ADT can be arbitrary. Next, we choose $\lambda^{*}=0.1$; then the switching law $\mathrm{S} 1$ will require

$$
\frac{T^{s}\left(t_{0}, t\right)}{T^{u}\left(t_{0}, t\right)} \geq \frac{\lambda_{u}+\lambda^{*}}{\lambda_{s}-\lambda^{*}}=\frac{5.1}{1.9} \approx 2.68 .
$$

According to Theorem 9, the switched system (49) is ISSiM under the above switching law S1.

To illustrate the correctness of the above conclusion, we carry out some simulation results with the following choices. Initial condition $\left[x_{1}(0), x_{2}(0)\right]=[-2.5,3]$, and switching path

$\sigma(t)$

$$
= \begin{cases}2, & t \in\left[t_{2 m}, t_{2 m+1}\right), t_{2 m+1}-t_{2 m}=0.2 * \text { rand, } \\ 1, & t \in\left[t_{2 m+1}, t_{2 m+2}\right), t_{2 m+2}-t_{2 m+1}=0.6+0.1 * \text { rand, }\end{cases}
$$




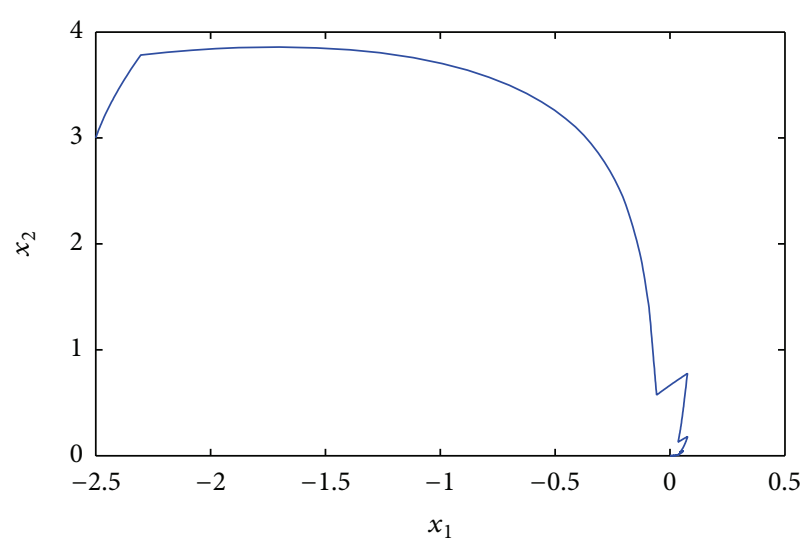

FIGURE 1: The state's response.

where $m=0,1,2, \ldots$, rand $\in(0,1)$ is a stochastic number. The simulation result is given in Figure 1, which is the response of the state under the above path $\sigma(t)$.

It can be observed from Figure 1 that the trajectory $x(t)$ converges to origin quickly. The simulation shows that Theorem 9 is very effective in analyzing the stability for the SSN systems with both unstable and ISSiM subsystems.

\section{Conclusions}

In this paper, we have investigated the ISSiM property of a class of SSN systems under ADT switching signals in two cases: (i) all of the constituent subsystems are ISSiM and (ii) parts of constituent subsystems are ISSiM and then proposed several new results about ISSiM of such systems. Firstly, a new ISSiM result for the SSN systems whose constituent subsystems are ISSiM has been obtained by applying an improved ADT method. Secondly, a new ISSiM result for the SSN system in which parts of subsystems are ISSiM has been given. In comparison with the existing results, the main results obtained in this paper have some advantages in some cases. Finally, an illustrative example with simulation has verified the validity and correctness of our results.

\section{Conflict of Interests}

The authors declare that they have no financial and personal relationships with other people or organizations that can inappropriately influence their work; there is no professional or other personal interest of any nature or kind in any product, service, and/or company that could be construed as influencing the position presented in, or the review of, this paper.

\section{Acknowledgments}

The paper was supported by the National Nature Science Foundation of China [61034007, 11226154, 61374074, 51107069, 61304133, and 61305130], China Postdoctoral Science Foundation funded Project [2013M541915 and 2013M541912], the Scientific Research Foundation of
Shandong province Outstanding Young Scientist Award [BS2013SF023], and the Natural Science Foundation of Shandong province [ZR2012AQ01 and, ZR2013FQ003].

\section{References}

[1] J. Daafouz, P. Riedinger, and C. Iung, "Stability analysis and control synthesis for switched systems: a switched Lyapunov function approach," IEEE Transactions on Automatic Control, vol. 47, no. 11, pp. 1883-1887, 2002.

[2] M. Goncalves, A. Megretski, and A. Dahleh, "Global analysis of piecewise linear systems using impact maps and surface Lyapunov functions," IEEE Transactions on Automatic Control, vol. 48, no. 12, pp. 2089-2106, 2003.

[3] P. Dayawansa and F. Martin, "A converse Lyapunov theorem for a class of dynamical systems which undergo switching," IEEE Transactions on Automatic Control, vol. 44, no. 4, pp. 751-760, 1999.

[4] D. Liberzon and R. Tempo, "Common Lyapunov functions and gradient algorithms," IEEE Transactions on Automatic Control, vol. 49, no. 6, pp. 990-994, 2004.

[5] H. Lin and J. Antsaklis, "Stability and stabilizability of switched linear systems: a survey of recent results," IEEE Transactions on Automatic Control, vol. 53, no. 1, pp. 1-15, 2008.

[6] X. Zhao and X. Liu, "Improved results on stability of continuous-time switched positive linea systems," Automatica, vol. 50, no. 2, pp. 614-621, 2014.

[7] X. Zhao, H. Liu, and J. Zhang, "Multiple-mode observer design for a class of switched linear systems linear systems," IEEE Transactions on Automation Science and Engineering, no. 99, pp. $1-9,2013$.

[8] X. Zhao, P. Shi, and L. Zhang, "Asynchronously switched control of a class of slowly switched linear systems," Systems \& Control Letters, vol. 61, no. 12, pp. 1151-1156, 2012.

[9] S. Branicky, "Multiple Lyapunov functions and other analysis tools for switched and hybrid systems," IEEE Transactions on Automatic Control, vol. 43, no. 4, pp. 475-482, 1998.

[10] R. Guo and Y. Wang, "Stability analysis for a class of switched linear systems," Asian Journal of Control, vol. 14, no. 3, pp. 817826, 2012.

[11] P. Zhao, W. Feng, and Y. Kang, "Stochastic input-to-state stability of switched stochastic nonlinear systems," Automatica, vol. 48, no. 10, pp. 2569-2576, 2012.

[12] W. Feng, J. Tian, and P. Zhao, "Stability analysis of switched stochastic systems," Automatica, vol. 47, no. 1, pp. 148-157, 2011.

[13] W. Ni, D. Cheng, and X. Hu, "Minimum dwell time for stability and stabilization of switched linear systems," in Proceedings of the 7th World Congress on Intelligent Control and Automation (WCICA '08), pp. 4109-4115, 2008.

[14] R. Guo, P. Zhao, and K. Li, "Input-to-state stability for a class of nonlinear switched systems by a new average dwell time method," in Proceeding of the 32th Chinese Control Conference, pp. 860-865, 2013.

[15] J. Hespanha and A. Morse, "Stability of switched systems with average dwell-time," in Proceedings of the 38th IEEE Conference on Decision \& Control (CDC '99), vol. 3, pp. 2655-2660, 1999.

[16] G. Zhai, B. Hu, K. Yasuda, and A. Michel, "Stability analysis of switched systems with stable and unstable subsystems: an average dwell time approach," in Proceedings of the American Control Conference, pp. 200-204, 2000. 
[17] X. Zhao, L. Zhang, P. Shi, and M. Liu, "Stability of switched positive linear systems with average dwell time switching," Automatica, vol. 48, no. 6, pp. 1132-1137, 2012.

[18] M. A. Müller and F. Allgöwer, "Improving performance in model predictive control: switching cost functionals under average dwell-time," Automatica, vol. 48, no. 2, pp. 402-409, 2012.

[19] E. Sontag and Y. Wang, "On characterizations of the input-tostate stability property," Systems and Control Letters, vol. 24, no. 5, pp. 351-359, 1995.

[20] E. Sontag, "Comments on integral variants of ISS," Systems \& Control Letters, vol. 34, no. 1-2, pp. 93-100, 1998.

[21] Z. Jiang and Y. Wang, "Input-to-state stability for discrete-time nonlinear systems," Automatica, vol. 37, no. 6, pp. 857-869, 2001.

[22] N. Yeganefar, P. Pepe, and M. Dambrine, "Input-to-state stability of time-delay systems: a link with exponential stability," IEEE Transactions on Automatic Control, vol. 53, no. 6, pp. 1526-1531, 2008.

[23] W. Chen and W. Zheng, "Input-to-state stability and integral input-to-state stability of nonlinear impulsive systems with delays," Automatica, vol. 45, no. 6, pp. 1481-1488, 2009.

[24] W. Xie, C. Wen, and Z. Li, "Input-to-state stabilization of switched nonlinear systems," IEEE Transactions on Automatic Control, vol. 46, no. 7, pp. 1111-1116, 2001.

[25] L. Vu, D. Chatterjee, and D. Liberzon, "Input-to-state stability of switched systems and switching adaptive control," Automatica, vol. 43, no. 4, pp. 639-646, 2007.

[26] P. Zhao, W. Feng, and Y. Kang, "Stochastic input-to-state stability of switched stochastic nonlinear systems," Automatica, vol. 48, no. 10, pp. 2569-2576, 2012.

[27] P. Zhao and Z. Wang, "Input-to-state stability of switched stochastic nonlinear systems," in Proceedings of the 30th Chinese Control Conference (CCC '11), pp. 1404-1406, 2011.

[28] R. Guo, P. Zhao, and C. Zhang, "Stability for a class of switched stochastic continuous-time nonlinear systems by animproved average dwell time method," . In press. 


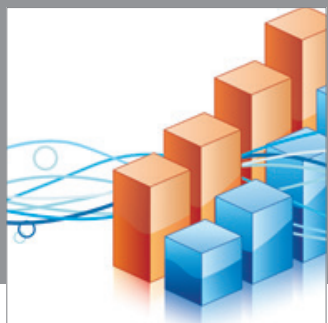

Advances in

Operations Research

mansans

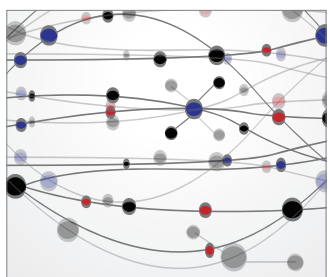

The Scientific World Journal
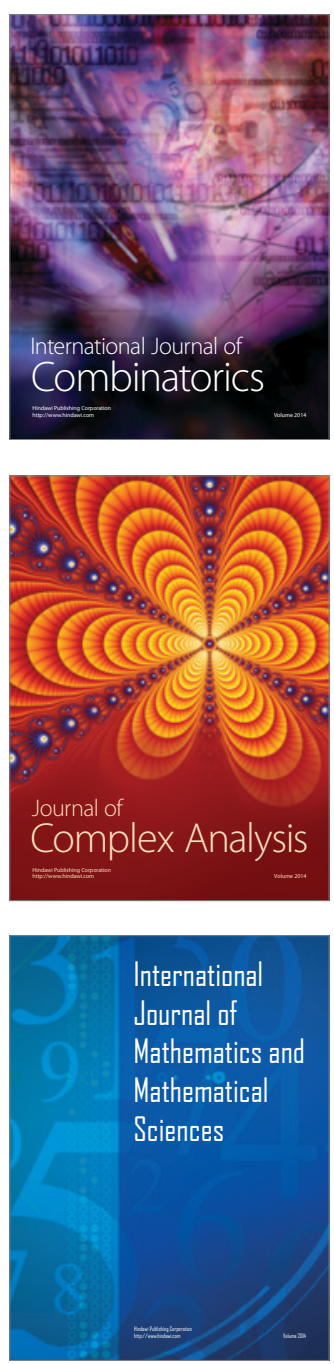
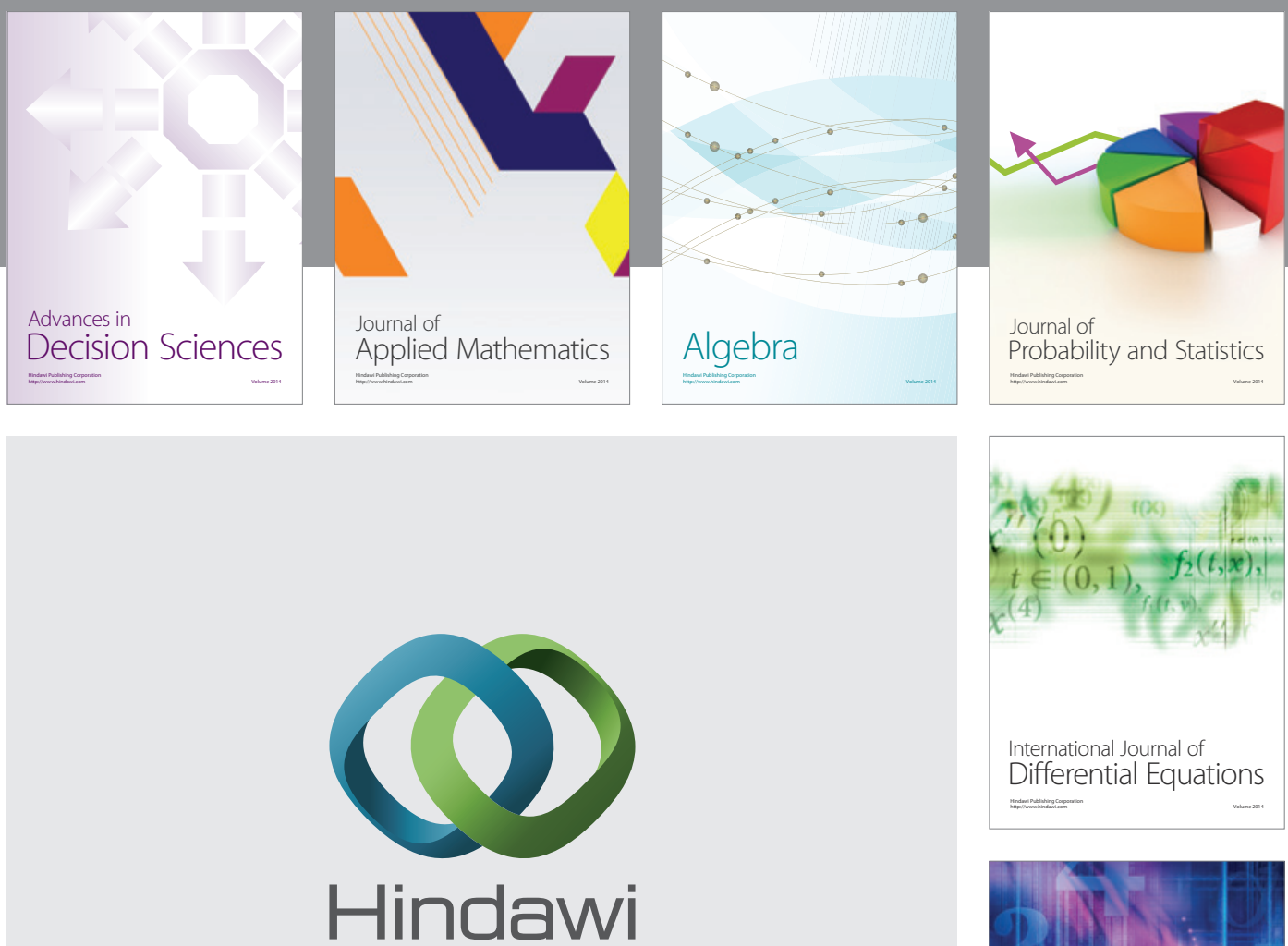

Submit your manuscripts at http://www.hindawi.com
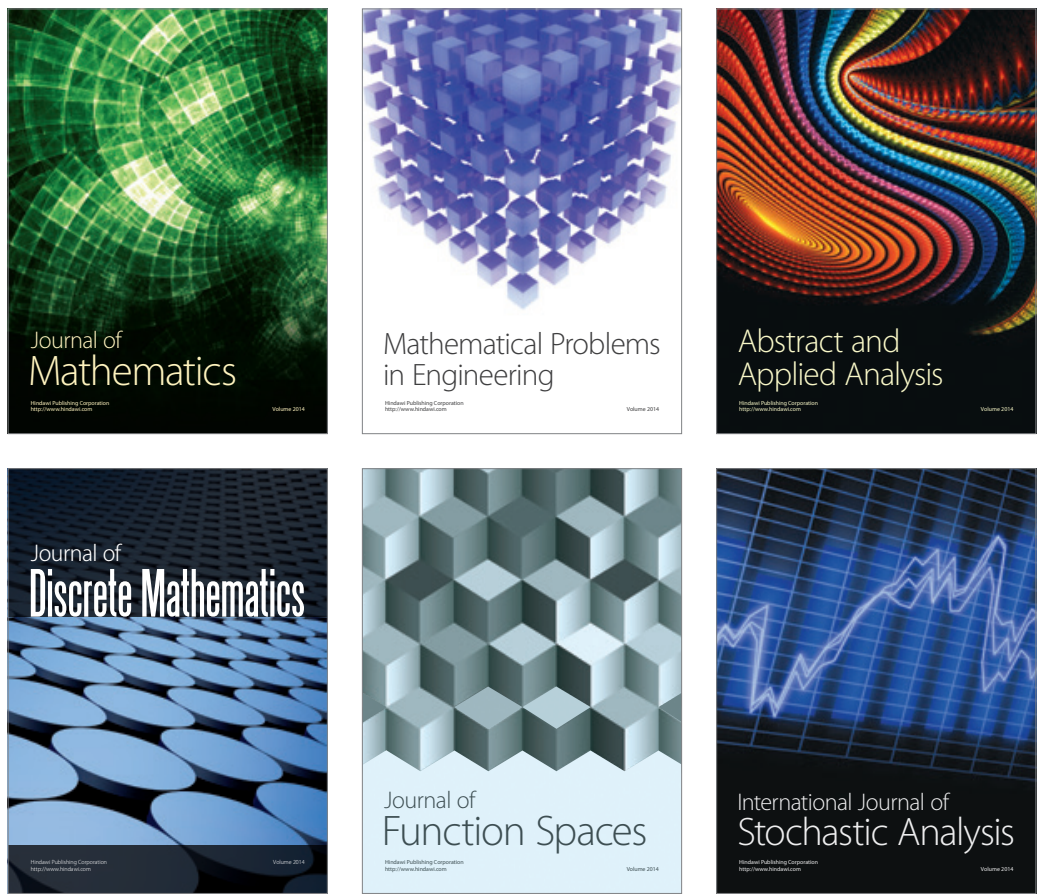

Journal of

Function Spaces

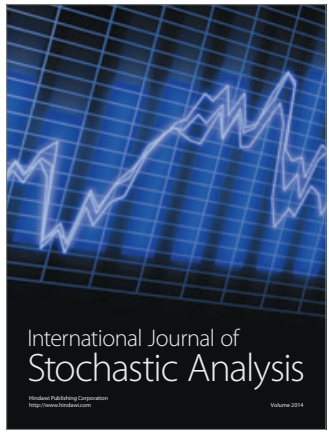

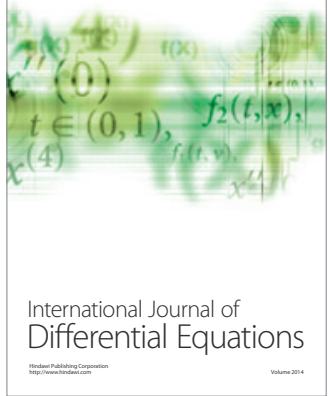
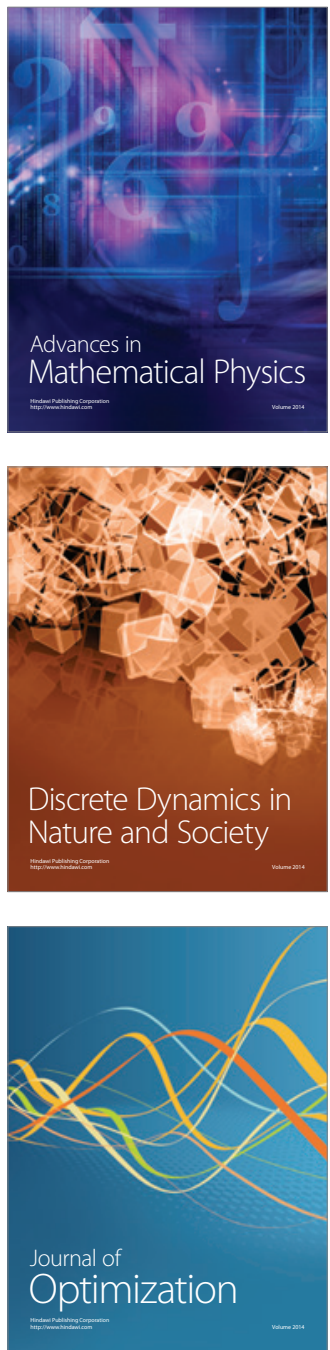